\title{
Shock Index on Admission Is Associated with Coronary Slow/No Reflow in Patients with Acute Myocardial Infarction Undergoing Emergent Percutaneous Coronary Intervention
}

\author{
Qingcheng Wang, ${ }^{1}$ Huimin Shen, ${ }^{1}$ Huijuan Mao, ${ }^{1}$ Fenghua Yu, \\ Haiqing Wang, ${ }^{2}$ and Jianlei Zheng $\mathbb{D}^{2}$ \\ ${ }^{1}$ Department of Cardiology, Hangzhou Yuhang Hospital of Traditional Chinese Medicine, Yuhang 311106, Zhejiang, China \\ ${ }^{2}$ Department of Cardiology, Zhejiang Provincial People's Hospital, People's Hospital of Hangzhou Medical College, \\ Hangzhou 310014, Zhejiang, China \\ Correspondence should be addressed to Jianlei Zheng; zhengjianlei@zjheart.com
}

Received 15 March 2019; Revised 6 May 2019; Accepted 12 June 2019; Published 25 July 2019

Academic Editor: Matteo Tebaldi

Copyright ( 2019 Qingcheng Wang et al. This is an open access article distributed under the Creative Commons Attribution License, which permits unrestricted use, distribution, and reproduction in any medium, provided the original work is properly cited.

\begin{abstract}
Objective. Coronary slow/no reflow is not rare after successfully undergoing primary percutaneous coronary intervention (PCI) in patients with acute myocardial infarction (AMI), and shock index (SI) is an important factor for adverse cardiovascular prognosis. In this study, we are to explore whether SI is associated with coronary slow/no reflow in patients with AMI following primary PCI. Methods. A total of 153 consecutive AMI patients undergoing primary PCI within 24 hours of symptom onset were included in this study. The participants were divided into normal flow group ( $n=124)$ and slow/no reflow group ( $n=29)$ according to cineangiograms recorded during the period of PCI. Cardiovascular risk factors, hematologic parameters, preoperative management of antithrombotic therapy, and baseline angiography were collected. Results. SI, plasma glucose, white blood cells (WBC) and neutrophil count, neutrophil to lymphocyte ratio (PLR), high sensitivity C-reactive protein (hs-CRP), probrain natriuretic peptide (pro-BNP), and Killip classification on admission and thrombus burden on initial angiography were significantly different between patients with and without slow/no reflow. Multivariate analysis revealed that $S I \geq 0.66$, thrombus burden, and plasma glucose on admission were independent predictors for coronary slow/no reflow. Preoperative management of tirofiban therapy improves initial thrombolysis in myocardial infarction (TIMI). However, it has no effect on prognosis of slow/no reflow. Conclusion. Our findings demonstrated that slow/no reflow in patients with AMI following primary PCI was more likely associated with $\mathrm{SI} \geq 0.66$, thrombus burden, and plasma glucose on admission. SI as a predictor for coronary slow/no reflow should be further confirmed in the following more large-scale and prospective studies. The clinical registration number is ChiCTR1900024447.
\end{abstract}

\section{Introduction}

By the virtue of primary percutaneous coronary intervention (PCI) with stenting implantation and combination with the dual antiplatelet drugs and statins therapy, the short- and long-term mortality of patients with acute myocardial infarction (AMI) were significantly decreased [1-3]. However, the incidence of coronary slow/no reflow was as high as $20 \%$ to $30 \%$ according to previous literature, so patients continued to suffer from severe impairment of myocardial reperfusion due to coronary slow/no reflow after successful opening of infarct related artery (IRA) $[4,5]$. The slow/no reflow commonly means the microvascular obstruction in distal coronary artery and is regarded as a predictor for adverse cardiovascular events $[6,7]$.

The pathogenesis of slow/no reflow phenomenon is sophisticated and has not been completely clarified up to now, but several hypotheses have been mentioned including distal microembolization of thrombus fragments, swelling of endothelial cells caused by ischemic and reperfusion injury, and microvascular spasm $[8,9]$. In clinical practices, a great number of studies have been carried out to investigate the 
predictors for slow/no reflow phenomenon, and the results showed that thrombosis burden, reperfusion time, inflammatory factors, the ratio of stent size to vessel diameter, and prehospital treatment with tirofiban were potentially associated with slow/no reflow and clinical prognosis in patients with AMI following emergent PCI [10-15]. However, shock index (SI) was an important factor for main adverse cardiovascular events (MACEs) in patients with AMI $[16,17]$, but it has been rarely reported in slow/no reflow phenomenon.

In consideration of the phenomenon of slow/no reflow as a severe complication of catheterization laboratory and a pivotal indicator for clinical outcomes, the aim of this study is to explore whether SI, preoperative use of tirofiban, hematological paremeters, cardiovascular risk factors, and initial angiographic features effectively foresee angiographic slow/no reflow in patients with AMI after primary PCI.

\section{Methods}

2.1. Study Population. Between November 2016 and July 2018, a total of 153 consecutive patients with acute myocardial infarction included ST elevation myocardial infarction (STEMI) and non-ST elevation myocardial infarction (NonSTEMI). All the patients underwent PCI with at least one drug-eluting stent. The diagnosis of STEMI was based on the following criteria: typical ischemic chest pain that lasted more than $30 \mathrm{~min}$ and ST-segment elevation $>1 \mathrm{~mm}$ in at least two contiguous leads or presumably new left bundle branch block on electrocardiogram combined with increased cardiac-specific biomarkers. In the absence of ST-segment elevation electrocardiogram, patients meeting the inclusion criteria were considered to have Non-STEMI. The patients with a history of recent surgery or trauma within the preceding 1 month and those with hematologic diseases, malignant tumors, febrile disorders, severe renal failure and hepatic dysfunction, and acute or chronic inflammatory disease on study entry were excluded. Besides, patients with AMI onset $\geq 24$ hours were not enrolled either in this study. All patients were provided with written informed consent. The study protocol conformed to the ethical guidelines of the Declaration of Helsinki and was approved by the ethical committees of Zhejiang Provincial People's Hospital.

\subsection{Study Protocol and Definitions. Coronary angiography} and PCI were performed through radial access after symptom onset within 24 hours. The IRA was the only target of emergent PCI. Angiographic slow/no reflow during PCI was defined as TIMI flow grade $\leq 2$ during the procedure without evidence of dissection, residual stenosis, distal embolism, or vasospasm. The TIMI flow grades were determined by the consensus of 2 intervention clinicians. Blood samples for measuring hematologic parameters, hs-CRP, pro-BNP, cardiac troponin I (cTNI), serum creatinine, blood urea nitrogen, and glucose were collected upon admission from a peripheral vessel in the emergency unit. Patients were given aspirin $300 \mathrm{mg}$ and clopidogrel $300 \mathrm{mg}$ as a loading dose before PCI. Then $100 \mathrm{mg}$ of aspirin combined with $75 \mathrm{mg}$ of clopidogrel once per day or $90 \mathrm{mg}$ of ticagrelor twice per day was given after PCI. In addition, 10-15 ml tirofiban (100ml/5mg, Grand Pharmaceutical Co., Ltd., China) was given to patients with AMI onset $\geq 3$ hours and accompanied with consistent angina in the emergency department. The angiographic morphologic features of burden thrombus in the IRA are scored in five degrees according to Gibson [18]: high burden thrombus formation if the TIMI thrombus was more than class 3. Multivessel coronary artery disease was defined as significant stenosis (coronary stenosis $\geq 70 \%$ ) of more than one epicardial coronary artery, including the culprit artery. Killip classification was introduced according to previous literature [19]. SI was defined as the ratio of heart rate to systolic blood pressure. In this study, the value of SI was measured in the emergency department. It was reported that $\mathrm{SI} \geq 0.66$ was a strong predictor for MACEs in patients with AMI [16]. Hence, we further classified value of SI into $\geq 0.66$ and $<0.66$. Clinical information of in-hospital outcomes included cardiac death for any reason, nonfatal reinfarction, ventricular tachycardia and/or ventricular fibrillation, and severe cardiac failure. Nonfatal reinfarction was defined as recurrent clinical angina symptoms with electrocardiogram changes compatible with MI or cTNI level at least twice the upper limit of normal range [20]. Severe cardiac failure was defined as congestive heart failure and/or cardiogenic shock that required treatment.

2.3. Statistical Analysis. Continuous variables are expressed as mean \pm standard deviation (SD), and categorical data are presented as percentages. Differences in continuous variables between two groups were assessed by unpaired 2-tailed ttest. Categorical data and proportions were analyzed by chisquare test. Univariate and multivariate analyses were performed to identify predictors of slow/no reflow phenomenon. Receiver operating characteristic (ROC) analysis was used to evaluate the sensitivity and specificity of SI predicting slow/no reflow. All $\mathrm{p}<0.05$ were considered statistically significant. Analyses were done with statistical software SPSS 11.0 (SPSS, Inc., Chicago, IL).

\section{Results}

3.1. Clinical and Angiographic Characteristics. Among the 153 patients with AMI who underwent primary PCI, 29 patients underwent angiographic slow/no reflow during PCI. The prevalence of high thrombus burden $(p=0.001)$ and inhospital outcomes $(\mathrm{p}<0.001)$ were higher in patients with slow/no reflow than those with normal reflow. The SI was significantly different between patients with slow/no reflow and normal reflow $(p=0.003)$. As a result, we found that the ratio of $\mathrm{SI} \geq 0.66$ was much higher in slow/no reflow than that in normal reflow $(\mathrm{p}=0.001)$. Compared with the normal reflow group, the ratio of Killip classes 3 or 4 was higher in slow/no reflow group $(\mathrm{p}=0.034)$. Left ventricular ejection fraction (LVEF) after PCI was lower in patients with slow/no reflow ( $\mathrm{p}=0.024)$, compared with patients with normal reflow. There was a definite association between preoperative usage of glycoprotein IIb/IIIa inhibitors, tirofiban, and initial TIMI level (Pearson's $\mathrm{r}=0.189, p=0.019$ ), but preoperative treatment 


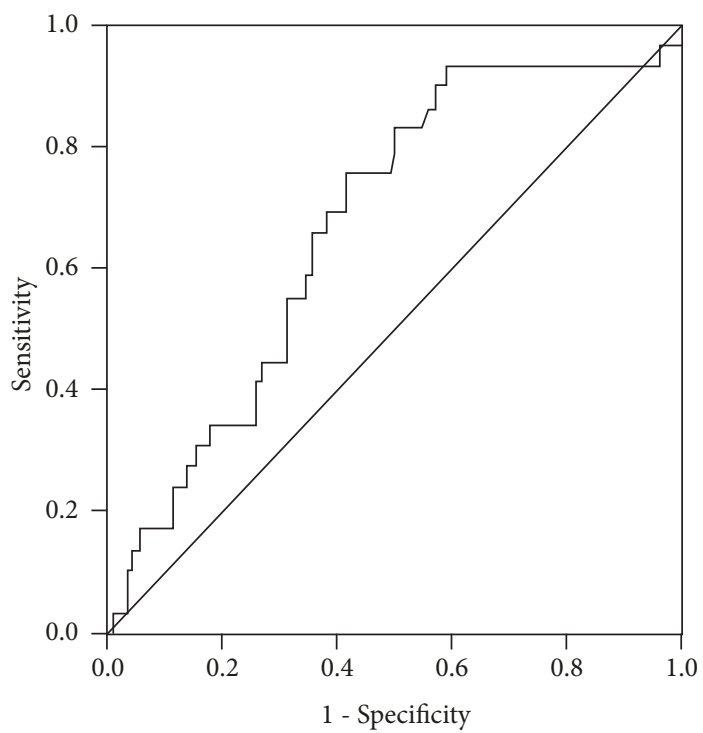

FIGURE 1: Receiver operating characteristic analysis displaying the power of shock index in the prediction of slow/no reflow phenomenon.

of tirofiban did not decrease the occurrence of slow/no reflow. The distribution of infarct-related coronary artery and cardiovascular risk factors including hypertension, hyperlipidemia, diabetes mellitus, and in-hospital medication were similar between these two groups (Table 1). In addition, the stent diameter and length, maximal balloon pressure, and aspiration ratio between these two groups were also similar.

3.2. Preoperative Laboratory Data of Patients. Plasma glucose $(\mathrm{p}<0.001)$, White blood cells $(\mathrm{p}=0.011)$, neutrophil counts $(\mathrm{p}=0.002)$, neutrophil to lymphocyte ratio (NLR) $(\mathrm{p}=0.012)$, hs-CRP $(\mathrm{p}=0.045)$, and pro-BNP $(\mathrm{p}=0.041)$ on admission were higher in patients with slow/no reflow than those with normal reflow. Other hematological parameters such as hemoglobin, platelet count, mean platelet volume (MPV), platelet distribution width (PDW), and platelets to lymphocytes ratio (PLR) were similar between these two groups. Furthermore, the levels of serum cTNI, creatinine, and urea nitrogen were of no statistical significance between patients with slow/no reflow and normal reflow (Table 2).

3.3. Predictors for Angiographic Slow/No Reflow. Univariate analysis showed that $S I \geq 0.66(p=0.001)$, high thrombus burden $(\mathrm{p}=0.002)$, Killip classes $3 / 4(\mathrm{p}=0.040)$, plasma glucose $(\mathrm{p}<0.001)$, White blood cells $(\mathrm{p}=0.014)$, neutrophil counts $(\mathrm{p}=0.004)$, neutrophil to lymphocyte ratio (NLR) $(\mathrm{p}=0.019)$, and hs-CRP $(p=0.009)$ on admission were associated with lesion progression. After adjustment for age, sex, and smoking, multivariate logistic regression analysis revealed that $\mathrm{SI} \geq 0.66(\mathrm{OR}=3.645,95 \% \mathrm{CI}=1.206-11.018$, and $\mathrm{p}=0.022)$, high thrombus burden $(\mathrm{OR}=3.536,95 \% \mathrm{CI}=1.324-9.438$, and $\mathrm{p}=0.031)$, and plasma glucose $(\mathrm{OR}=1.116,95 \% \mathrm{CI}=1.010-1.232$, and $\mathrm{p}=0.012$ ) were still independent determinants for coronary slow/no reflow in patients with AMI undergoing emergent PCI (Table 3). ROC curve analysis revealed that the sensitivity was $76 \%$ and specificity was $59 \%$ for $\mathrm{SI}=0.66$ to predict slow/no reflow phenomenon, and the area under the curve of SI was 0.672 (CI 95\% 0.568-0.776, $p=0.004$ ) (Figure 1).

\section{Discussion}

The slow/no reflow phenomenon is frequently observed during the PCI, which can counteract partial benefits from catheter intervention. Although the exact mechanism of slow/no reflow was not fully clarified, inflammation, endothelial dysfunction, changes in blood components, reperfusion time, and angiographic characteristics of coronary lesions have been proposed in the pathogenesis of slow/no reflow $[10-15,21]$. In this study, we found that independent predictors of angiographic slow/no reflow during the emergent PCI were $\mathrm{SI} \geq 0.66$, plasma glucose on admission, and high thrombus burden on initial angiography.

In our current investigation, we found that high thrombus burden was associated with the phenomenon of slow/no reflow. In fact, the thrombus burden as a strong indicator for slow/no reflow has been reported in many studies [22, 23]. High thrombus burden was usually accompanied with the extended time to reperfusion, since delayed reperfusion leads to thrombus gathering more and more erythrocytes and becoming more steady; more importantly, red thrombi tend to form fragments and lead to distal embolization during the balloon dilatation $[7,24]$. As a result, microcirculatory dysfunction aggravates myocardial reperfusion injury and is associated with a higher risk to slow/no reflow and adverse cardiac events. Intensified antiplatelet treatment significantly improves platelet aggregation and inhibits thrombosis formation [25]. In our study, the rate of preoperative use of tirofiban was similar between two groups. The use of tirofiban was not an independent indicator for slow/no reflow, which significantly improves the initial TIMI. The effects of preoperative 
TABLE 1: Clinical and coronary angiographic features in patients with normal flow and slow/no reflow.

\begin{tabular}{|c|c|c|c|}
\hline & $\begin{array}{c}\text { Normal flow group } \\
\mathrm{n}=124\end{array}$ & $\begin{array}{c}\text { Slow/no reflow group } \\
n=29\end{array}$ & $P$ value \\
\hline Male gender, n (\%) & $96(77.4)$ & $25(86.2)$ & 0.295 \\
\hline Age, years & $63 \pm 14$ & $61 \pm 13$ & 0.423 \\
\hline Cigarette smoking, n (\%) & $60(48.4)$ & $16(55.2)$ & 0.511 \\
\hline Heart rate & $83 \pm 13$ & $86 \pm 14$ & 0.248 \\
\hline Systolic blood pressure, mmHg & $129 \pm 20$ & $123 \pm 18$ & 0.159 \\
\hline Diastolic blood pressure, $\mathrm{mmHg}$ & $80 \pm 14$ & $84 \pm 19$ & 0.306 \\
\hline Shock index & $0.65 \pm 0.09$ & $0.71 \pm 0.09$ & 0.003 \\
\hline Shock index $\geq 0.66, \mathrm{n}(\%)$ & $51(41.1)$ & $22(75.9)$ & 0.001 \\
\hline Diabetes mellitus, n (\%) & $34(27.4)$ & $8(27.6)$ & 0.986 \\
\hline Hypertension, n (\%) & $65(52.4)$ & $16(55.2)$ & 0.789 \\
\hline Hyperlipidemia, n (\%) & $31(25.0)$ & $10(34.5)$ & 0.299 \\
\hline Previous cerebrovascular disease, $\mathrm{n}(\%)$ & $15(12.1)$ & $5(17.2)$ & 0.459 \\
\hline Time to reperfusion (hour) & $7.63 \pm 5.90$ & $7.52 \pm 5.65$ & 0.926 \\
\hline STEMI, n (\%) & $84(67.7)$ & $21(72.4)$ & 0.625 \\
\hline Initial TIMI flow 0/1 grade, $\mathrm{n}(\%)$ & $95(76.6)$ & $26(89.7)$ & 0.120 \\
\hline High thrombus burden, n (\%) & $44(35.5)$ & $20(69.0)$ & 0.001 \\
\hline Preoperative use of tirofiban, $\mathrm{n}(\%)$ & $75(60.5)$ & $17(58.6)$ & 0.854 \\
\hline Aspiration, $\mathrm{n}(\%)$ & $24(19.4)$ & $6(20.7)$ & 0.871 \\
\hline Infarct-related coronary artery & & & 0.357 \\
\hline LAD, n (\%) & $62(50.0)$ & $15(51.7)$ & \\
\hline RCA, n (\%) & $41(33.1)$ & $12(41.4)$ & \\
\hline LCA, n (\%) & $21(16.9)$ & $2(6.9)$ & \\
\hline Killip classification & & & 0.034 \\
\hline $1 / 2, \mathrm{n}(\%)$ & $112(90.3)$ & $22(75.9)$ & \\
\hline $3 / 4, \mathrm{n}(\%)$ & $12(9.7)$ & $7(24.1)$ & \\
\hline Multivessel disease, $\mathrm{n}(\%)$ & $60(48.4)$ & $15(51.7)$ & 0.746 \\
\hline Stent diameter, mm & $3.07 \pm 0.30$ & $3.17 \pm 0.35$ & 0.100 \\
\hline Stent length, mm & $35 \pm 15$ & $34 \pm 17$ & 0.677 \\
\hline Maximal balloon pressure, atm & $13 \pm 3$ & $14 \pm 3$ & 0.085 \\
\hline LVEF after PCI, \% & $54 \pm 9$ & $49 \pm 8$ & 0.024 \\
\hline \multicolumn{4}{|l|}{ In-hospital medication } \\
\hline Aspirin, n (\%) & $123(99.2)$ & $28(96.6)$ & 0.259 \\
\hline Clopidogrel or ticagrelor, n (\%) & 123(99.2) & $28(96.6)$ & 0.259 \\
\hline ACEI or ARB, n (\%) & $74(59.7)$ & $17(58.6)$ & 0.917 \\
\hline$\beta$-Blockers, $\mathrm{n}(\%)$ & $54(43.5)$ & $10(34.5)$ & 0.373 \\
\hline Statins, n (\%) & $118(95.2)$ & $27(93.1)$ & 0.654 \\
\hline In-hospital outcomes, n (\%) & $2(1.6)$ & $5(17.2)$ & $<0.001$ \\
\hline
\end{tabular}

STEMI: ST-elevation myocardial infarction; TIMI: thrombolysis in myocardial infarction; LAD: left anterior descending artery; RCA: right coronary artery; LCA: left circumflex artery; LVEF: left ventricular ejection fraction; PCI: percutaneous coronary intervention; ACEI: angiotensin converting enzyme inhibitor; ARB: angiotensin-receptor blocker.

treatment with tirofiban on the prognosis of acute coronary syndrome (ACS) patients were extensively investigated in the past two decades, but the results were inconsistent $[15,26]$. Skyschally et al. reported that distal coronary embolization was associated with severe regional contractile dysfunction in animal model [27]; we also found that the value of LVEF after PCI was remarkably lower in patients with slow/no reflow than that in normal reflow. It is well known that LVEF was an independent predictor for adverse prognosis in patients with ACS [28].
Higher heart rate on admission was an important predictor for death or cardiac events in patients with ACS, and elevated resting heart rate was related to poor outcomes in heart failure with reduced ejection fraction and preserved ejection fraction $[29,30]$. In addition, it was mentioned that higher heart rate was associated with no reflow in patients with STEMI following primary PCI [22]. Low blood pressure is closely related to no reflow and higher in-hospital mortality and poorer cardiac function $[11,20]$. The concept of SI, defined as the ratio of heart rate to systolic blood 
TABLE 2: Preoperative laboratory data of patients.

\begin{tabular}{lccc}
\hline & Normal flow group & Slow/No reflow group & P value \\
& $\mathrm{n}=124$ & $12.52 \pm 6.86$ & $<0.001$ \\
\hline Plasma glucose, $\mathrm{mmol} / \mathrm{l}$ & $8.57 \pm 3.93$ & $11.71 \pm 2.63$ & 0.011 \\
WBC $\left(\times 10^{\wedge} 9 / \mathrm{L}\right)$ & $10.26 \pm 2.74$ & $9.97 \pm 2.97$ & 0.002 \\
neutrophil $\left(\times 10^{\wedge} 9 / \mathrm{L}\right)$ & $8.14 \pm 2.83$ & $1.22 \pm 0.83$ & 0.082 \\
lymphocyte $\left(\times 10^{\wedge} 9 / \mathrm{L}\right)$ & $1.58 \pm 1.01$ & $10.41 \pm 5.38$ & 0.012 \\
NLR & $7.24 \pm 6.20$ & $143 \pm 22$ & 0.868 \\
Hemoglobin $(\mathrm{g} / \mathrm{L})$ & $142 \pm 17$ & $192 \pm 51$ & 0.821 \\
PLT $\left(\times 10^{\wedge} 9 / \mathrm{L}\right)$ & $194 \pm 59$ & $190 \pm 94$ & 0.124 \\
PLR & $157 \pm 106$ & $10.16 \pm 1.47$ & 0.151 \\
MPV, fL & $9.77 \pm 1.28$ & $15.97 \pm 1.74$ & 0.086 \\
PDW $(\%)$ & $15.39 \pm 1.60$ & $21.46 \pm 19.89$ & 0.045 \\
hs-CRP, $\mathrm{mg} / \mathrm{l}$ & $13.43 \pm 12.19$ & $5.99 \pm 1.85$ & 0.501 \\
Blood urea nitrogen, mmol /L & $6.31 \pm 2.39$ & $83.87 \pm 20.22$ & 0.950 \\
Creatinine, $\mu \mathrm{mol} / \mathrm{L}$ & $83.51 \pm 28.34$ & $19.85 \pm 25.17$ & 0.465 \\
cTNI, $\mu \mathrm{g} / \mathrm{L}$ & $15.65 \pm 28.35$ & $769 \pm 940$ & 0.041 \\
pro-BNP, pg/mL & $438 \pm 736$ & & \\
\hline
\end{tabular}

Data are mean \pm SD.

WBC: white blood cell count; NLR: neutrophil to lymphocyte ratio; PLT: platelet count; PLR: platelets to lymphocytes ratio; MPV: mean platelet volume; PDW: platelet distribution width; hsCRP: high sensitivity C-reactive protein; cTNI: cardiac troponin I; pro-BNP: probrain natriuretic peptide.

TABLE 3: Multivariate analysis of risk factors for patients with normal flow and slow/no reflow.

\begin{tabular}{lccc}
\hline Variables & OR & $95 \%$ CI & p value \\
\hline Plasma glucose & 1.116 & $1.010-1.232$ & 0.031 \\
High thrombus burden & 3.536 & $1.324-9.438$ & 0.012 \\
Shock index $\geq 0.66$ & 3.645 & $1.206-11.018$ & 0.022 \\
\hline
\end{tabular}

OR: odds ratio; CI: confidence internal.

pressure, is a relatively objective indicator independently of systolic blood pressure and heart rate under excitement of sympathetic nerve [31], which cause a concurrent increase of heart rate and systolic blood pressure. Studies showed that SI was strongly associated with in-hospital mortality in patients with ACS following primary PCI, and $\mathrm{SI} \geq 0.66$ representing a cutoff value for clinical prediction was demonstrated in several studies $[16,17]$. In the current study, we found that patients with slow/no reflow had higher ratio of $\mathrm{SI} \geq 0.66$, compared with the normal reflow. After adjusting other risk factors, $S I \geq 0.66$ was still a pivotal predictor for slow/no reflow.

It has been mentioned that hyperglycemia was associated with an impairment of microvascular function and could cause angiographic slow/no reflow [32]. There are several mechanisms of hyperglycemia-associated angiographic slow/no reflow. Specially, hyperglycemia aggravates platelet-dependent thrombosis, inhibits endothelium dependent vasodilatation, and decreases collateral blood flow by decreasing nitric oxide availability [33-35]. In accordance with previous studies, multivariate logistic regression showed that the hyperglycemia on admission was an independent factor for slow/no reflow in our study.

Abnormality of hematologic parameters has also been implicated in the pathogenesis of the slow/no reflow phenomenon. Elevated WBC and NLR were reported as a risk factor for indicating systemic inflammatory response, and MPV as well as PDW was a potentially useful marker of platelet activity $[22,36]$. In our study, we found that WBC, neutrophil, NLR, and hs-CRP were notably increased in slow/no reflow group than those in normal group. However, these inflammatory indicators were not an independent predictor factor for slow/no reflow. We supposed that it was possibly associated with our relatively small samples. Moreover, above parameters as a powerful predictor should be further verified in other studies.

The process of PCI itself was related to PCI-related slow/no reflow, and it was demonstrated that the length of implanted stent and overexpansion of stent were associated with occurrence of no reflow $[37,38]$. The balloon or stent expansion crushes the plaques and causes the lipid core rupture; lipid fraction activates the thrombosis formation and fragment obstructs the distal microcirculation. It has been approved that there is a possible relationship between lipidrich plaque and no reflow via preinterventional optical coherence tomography examination [39]. In the current study, we observed that balloon dilatation pressures of stenting were slightly higher in slow/no reflow group than those in normal group. However, the stent length and diameter were similar between these two groups.

In conclusion, considering the complicated mechanism and severe adverse prognosis of slow/no reflow phenomenon, 
it is very meaningful to find more useful and powerful predictors to prejudge the occurrence of slow/no reflow. In this retrospective investigation, we observed that plasma glucose, $\mathrm{SI} \geq 0.66$ on admission, and high thrombus load on initial angiography were related to angiographic slow/no reflow phenomenon in patients with AMI following primary PCI. ROC curve analysis showed that the area under the curve of SI was 0.672 . We considered that more than $40 \%$ patients in normal flow had $S I \geq 0.66$, which possibly affected the power of SI predicting slow/no reflow phenomenon. In addition, we are conscious that the sample size in our study was small; thus some group comparisons may have lacked power to detect significant differences for selected variables. In addition, larger-scale studies should be performed to confirm the association between above predictors and slow/no reflow.

\section{Data Availability}

The data used to support the findings of this study are available from the corresponding author upon request.

\section{Conflicts of Interest}

The authors declare no conflicts of interest.

\section{Acknowledgments}

This work was supported by the Clinical Research Fund from Zhejiang Medical Association (No. 2017ZYC-B1) and the Natural Science Foundation of Zhejiang Province (No. LY17H020013).

\section{References}

[1] C. M. Yanamala, P. K. Bundhun, and A. Ahmed, "Comparing mortality between fibrinolysis and primary percutaneous coronary intervention in patients with acute myocardial infarction," Coronary Artery Disease, vol. 28, no. 4, pp. 315-325, 2017.

[2] C. Lee, C. Cheng, Y. K. Yang et al., "Trends in the incidence and management of acute myocardial infarction from 1999 to 2008: get with the guidelines performance measures in Taiwan," Journal of the American Heart Association, vol. 3, no. 4, 2014.

[3] K. Ishida, T. Geshi, A. Nakano et al., "Beneficial effects of statin treatment on coronary microvascular dysfunction and left ventricular remodeling in patients with acute myocardial infarction," International Journal of Cardiology, vol. 155, no. 3, pp. 442-447, 2012.

[4] S. H. Rezkalla, K. C. Dharmashankar, I. B. Abdalrahman, and R. A. Kloner, "No-reflow phenomenon following percutaneous coronary intervention for acute myocardial infarction: incidence, outcome, and effect of pharmacologic therapy," Journal of Interventional Cardiology, vol. 23, no. 5, pp. 429-436, 2010.

[5] I. Morishima, T. Sone, K. Okumura et al., "Angiographic no-reflow phenomenon as a predictor of adverse long-term outcome in patients treated with percutaneous transluminal coronary angioplasty for first acute myocardial infarction," Journal of the American College of Cardiology, vol. 36, no. 4, pp. 1202-1209, 2000.

[6] R. Jaffe, T. Charron, G. Puley, A. Dick, and B. H. Strauss, "Microvascular obstruction and the no-reflow phenomenon after percutaneous coronary intervention," Circulation, vol. 117, no. 24, pp. 3152-3156, 2008.

[7] L. Dong-bao, H. Qi, L. Zhi, W. Shan, and J. Wei-ying, "Predictors and long-term prognosis of angiographic slow/no-reflow phenomenon during emergency percutaneous coronary intervention for ST-elevated acute myocardial infarction," Clinical Cardiology, vol. 33, no. 12, pp. E7-E12, 2010.

[8] A. Karimianpour and A. Maran, "Advances in coronary no-reflow phenomenon-a contemporary review," Current Atherosclerosis Reports, vol. 20, no. 9, 2018.

[9] S. Gupta and M. M. Gupta, "No reflow phenomenon in percutaneous coronary interventions in ST-segment elevation myocardial infarction," Indian Heart Journal, vol. 68, no. 4, pp. 539-551, 2016.

[10] K. Wang, J. Zhang, N. Zhang et al., "Combined primary PCI with multiple thrombus burden reduction therapy improved cardiac function in patients with acute anterior myocardial infarction," International Heart Journal, vol. 60, no. 1, pp. 27-36, 2019.

[11] H. Zhou, X. Y. He, S. W. Zhuang et al., "Clinical and procedural predictors of no-reflow in patients with acute myocardial infarction after primary percutaneous coronary intervention," World Journal of Emergency Medicine, vol. 5, no. 2, pp. 96-102, 2014.

[12] J. Wang, Y. Chen, C. Wang, X. Yang, X. Zhu, and Z. Zhou, "Development and Validation of a clinical risk score predicting the no-reflow phenomenon in patients treated with primary percutaneous coronary intervention for ST-segment elevation myocardial infarction," Cardiology, vol. 124, no. 3, pp. 153-160, 2013.

[13] C. Bouleti, N. Mewton, and S. Germain, "The no-reflow phenomenon: state of the art," Archives of Cardiovascular Diseases, vol. 108, no. 12, pp. 661-674, 2015.

[14] Y. Watanabe, K. Sakakura, Y. Taniguchi et al., "Determinants of slow flow in percutaneous coronary intervention to the culprit lesion of non-ST elevation myocardial infarction," International Heart Journal, vol. 59, no. 6, pp. 1237-1245, 2018.

[15] E. Fabris, S. Kilic, D. A. Schellings et al., "Long-term mortality and prehospital tirofiban treatment in patients with ST elevation myocardial infarction," Heart, vol. 103, no. 19, pp. 1515-1520, 2017.

[16] N. Abe, T. Miura, Y. Miyashita et al., "Long-term prognostic implications of the admission shock index in patients with acute myocardial infarction who received percutaneous coronary intervention," Angiology, vol. 68, no. 4, pp. 339-345, 2016.

[17] X. Zhang, Z. Wang, Z. Wang, M. Fang, and Z. Shu, "The prognostic value of shock index for the outcomes of acute myocardial infarction patients," Medicine, vol. 96, no. 38, p. e8014, 2017.

[18] C. M. Gibson, J. A. de Lemos, S. A. Murphy et al., "Combination therapy with abciximab reduces angiographically evident thrombus in acute myocardial infarction: a TIMI 14 substudy," Circulation, vol. 103, no. 21, pp. 2550-2554, 2001.

[19] E. Taguchi, Y. Konami, M. Inoue et al., "Impact of Killip classification on acute myocardial infarction: data from the SAIKUMA registry," Heart and Vessels, vol. 32, no. 12, pp. 14391447, 2017.

[20] J. S. Park, K. S. Cha, D. Shin et al., "Prognostic significance of presenting blood pressure in patients with ST-elevation myocardial infarction undergoing percutaneous coronary intervention," American Journal of Hypertension, vol. 28, no. 6, pp. 797805, 2015. 
[21] J. Tian, Y. Liu, Y. Liu et al., "Prognostic association of circulating neutrophil count with no-reflow in patients with ST-segment elevation myocardial infarction following successful primary percutaneous intervention," Disease Markers, vol. 2017, Article ID 8458492, 9 pages, 2017.

[22] J. K. Fajar, T. Heriansyah, and M. S. Rohman, "The predictors of no reflow phenomenon after percutaneous coronary intervention in patients with ST elevation myocardial infarction: A meta-analysis," Indian Heart Journal, vol. 70, pp. S406-S418, 2018.

[23] I. H. Tanboga, S. Topcu, E. Aksakal, K. Kalkan, S. Sevimli, and M. Acikel, "Determinants of angiographic thrombus burden in patients with ST-segment elevation myocardial infarction," Clinical and Applied Thrombosis/Hemostasis, vol. 20, no. 7, pp. 716-722, 2014.

[24] W. J. van der Spuy and E. Pretorius, "Interaction of red blood cells adjacent to and within a thrombus in experimental cerebral ischaemia," Thrombosis Research, vol. 132, no. 6, pp. 718-723, 2013.

[25] X. Yang, M. A. Leesar, H. Ahmed et al., "Impact of ticagrelor and aspirin versus clopidogrel and aspirin in symptomatic patients with peripheral arterial disease: Thrombus burden assessed by optical coherence tomography," Cardiovascular Revascularization Medicine, vol. 19, no. 7, pp. 778-784, 2018.

[26] S. Chen, Y. Hsieh, G. B. Guo et al., "Angiographic and clinical outcome in ST-segment elevation myocardial infarction patients receiving an adjunctive double bolus regimen of tirofiban for primary percutaneous coronary intervention," Circulation Journal, vol. 70, no. 5, pp. 536-541, 2006.

[27] A. Skyschally, M. Haude, H. Dörge et al., "Glucocorticoid treatment prevents progressive myocardial dysfunction resulting from experimental coronary microembolization," Circulation, vol. 109, no. 19, pp. 2337-2342, 2004.

[28] D. Saito, R. Nakanishi, I. Watanabe et al., "Combined assessment of left ventricular end-diastolic pressure and ejection fraction by left ventriculography predicts long-term outcomes of patients with ST-segment elevation myocardial infarction," Heart and Vessels, vol. 33, no. 5, pp. 453-461, 2018.

[29] X. Shang, R. Lu, M. Liu, S. Xiao, and N. Dong, "Heart rate and outcomes in patients with heart failure with preserved ejection fraction," Medicine, vol. 96, no. 43, p. e8431, 2017.

[30] T. S. Garadah, K. B. Thani, L. Sulibech, A. A. Jaradat, M. E. Al Alawi, and H. Amin, "Risk stratification and in hospital morality in patients presenting with acute coronary syndrome (ACS) in Bahrain," The Open Cardiovascular Medicine Journal, vol. 12, no. 1, pp. 7-17, 2018.

[31] A. S. Keller, L. L. Kirkland, S. Y. Rajasekaran, S. Cha, M. Y. Rady, and J. M. Huddleston, "Unplanned transfers to the intensive care unit: The role of the shock index," Journal of Hospital Medicine, vol. 5, no. 8, pp. 460-465, 2010.

[32] A. Yildiz, A. Arat-Ozkan, C. Kocas et al., "Admission hyperglycemia and TIMI frame count in primary percutaneous coronary intervention," Angiology, vol. 63, no. 5, pp. 325-329, 2011.

[33] M. Shechter, C. N. B. Merz, M. J. Paul-Labrador, and S. Kaul, "Blood glucose and platelet-dependent thrombosis in patients with coronary artery disease," Journal of the American College of Cardiology, vol. 35, no. 2, pp. 300-307, 2000.

[34] L. M. Title, P. M. Cummings, K. Giddens, and B. A. Nassar, "Oral glucose loading acutely attenuates endotheliumdependent vasodilation in healthy adults without diabetes: an effect prevented by vitamins C and E," Journal of the American College of Cardiology, vol. 36, no. 7, pp. 2185-2191, 2000.

[35] J. R. Kersten, W. G. Toller, J. P. Tessmer, P. S. Pagel, and D. C. Warltier, "Hyperglycemia reduces coronary collateral blood flow through a nitric oxide-mediated mechanism," American Journal of Physiology-Heart and Circulatory Physiology, vol. 281, no. 5, pp. H2097-H2104, 2001.

[36] T. Celık, S. Balta, M. Demir et al., "Predictive value of admission red cell distribution width-platelet ratio for no-reflow phenomenon in acute ST segment elevation myocardial infarction undergoing primary percutaneous coronary intervention," Cardiology Journal, vol. 23, no. 1, pp. 84-92, 2016.

[37] Y. Maekawa, Y. Asakura, T. Anzai et al., "Relation of stent overexpansion to the angiographic no-reflow phenomenon in intravascular ultrasound-guided stent implantation for acute myocardial infarction," Heart and Vessels, vol. 20, no. 1, pp. 1318, 2005.

[38] A. Bayramoglu, H. Tasolar, A. Kaya et al., "Prediction of noreflow and major adverse cardiovascular events with a new scoring system in STEMI patients," Journal of Interventional Cardiology, vol. 31, no. 2, pp. 144-149, 2018.

[39] A. Tanaka, T. Imanishi, H. Kitabata et al., "Lipid-rich plaque and myocardial perfusion after successful stenting in patients with non-ST-segment elevation acute coronary syndrome: an optical coherence tomography study," European Heart Journal, vol. 30, no. 11, pp. 1348-1355, 2009. 


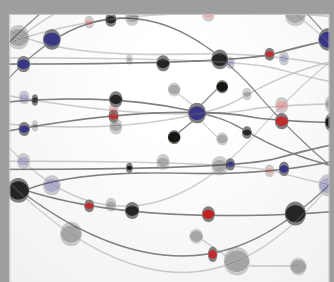

The Scientific World Journal
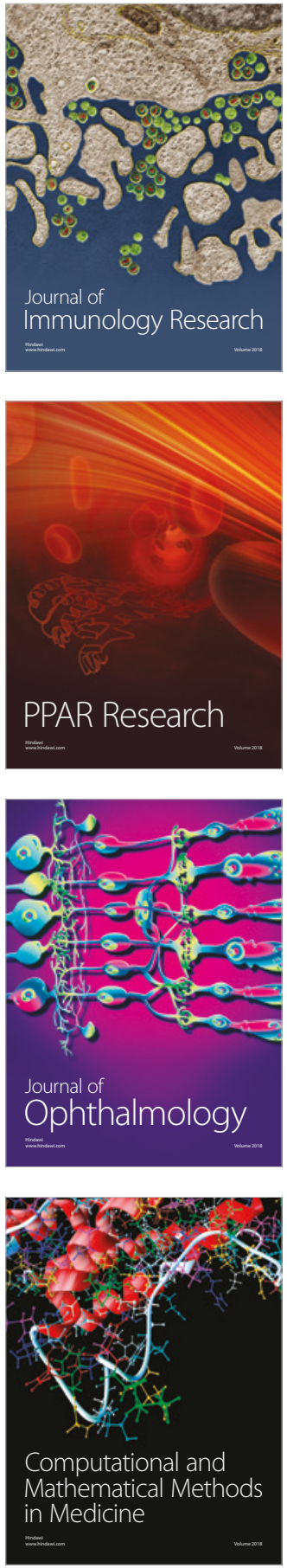

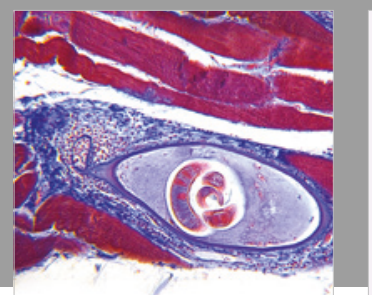

Gastroenterology Research and Practice

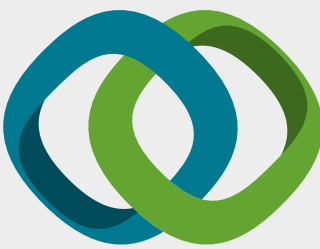

\section{Hindawi}

Submit your manuscripts at

www.hindawi.com
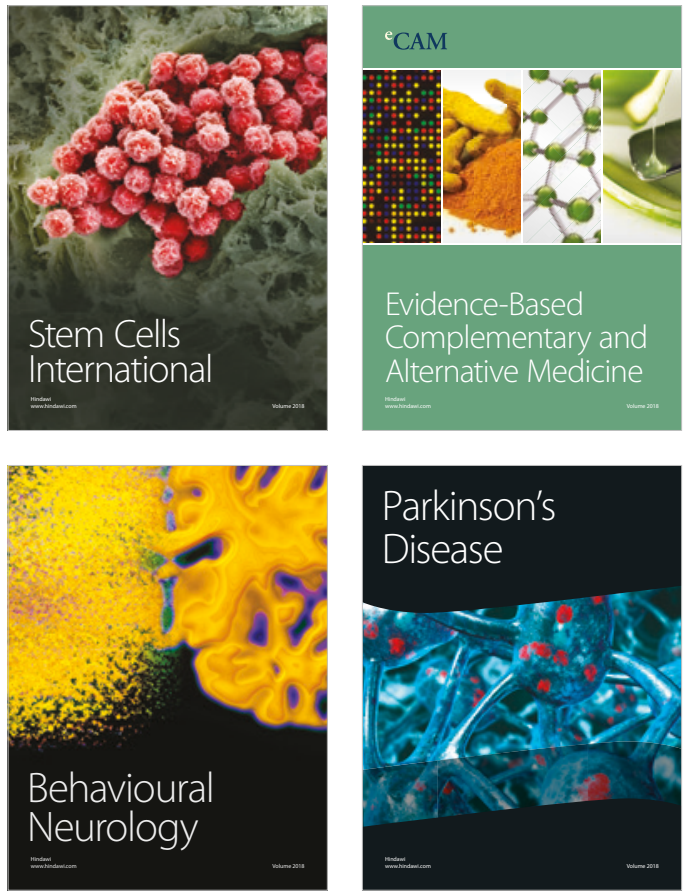

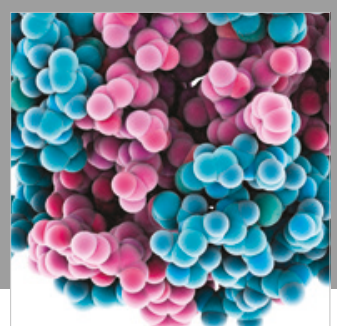

ournal of

Diabetes Research

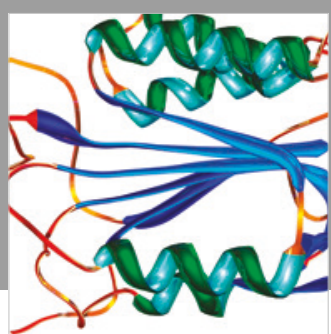

Disease Markers
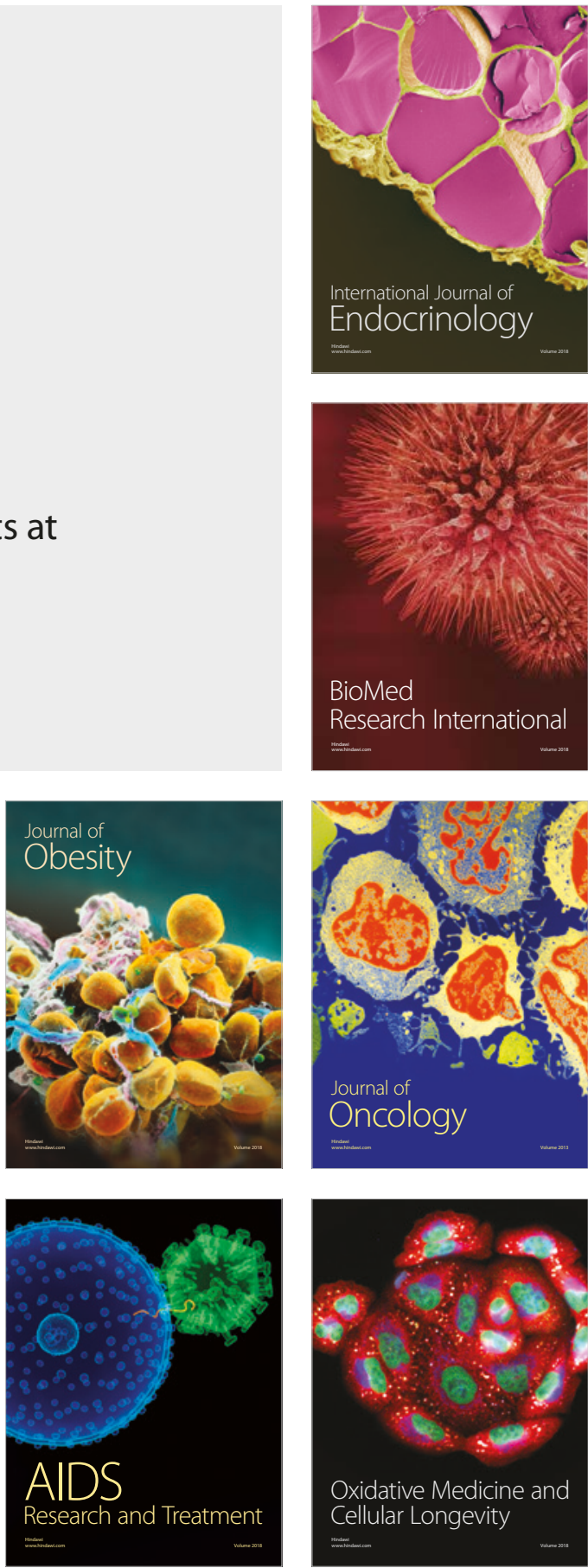